\title{
Expression of TLR4-PTGE2 signaling genes in atherosclerotic carotid plaques and peripheral blood.
}

Ferronato $\mathrm{S}^{1}$, Scuro $\mathrm{A}^{2}$, Fochi $\mathrm{S}^{1}$, Orlandi $\mathrm{E}^{1}$, Gomez-Lira $\mathrm{M}^{1}$, Olivato $\mathrm{S}^{3}$, Mazzucco $\mathrm{S}^{4}$, Turco $\mathrm{A}^{1}$, Romanelli $\mathrm{MG}^{1}$

Silvia Ferronato:0000-0003-1103-3089

Silvia Olivato: 0000-0002-7052-4155

Sara mazzucco: 0000-0002-1582-6724

Elisa Orlandi: 0000-0002-1484-4170

Maria Grazia Romanelli: 0000-0002-7360-1195

Alberto Turco: 0000-0002-3476-7694

Alberto Scuro: 0000-0002-7986-8624

Macarena Gomez-Lira: 0000-0003-4119-2267

Stefania Fochi: 0000-0002-8616-5775

\section{Institutional addresses (affiliations):}

${ }^{1}$ Department of Neurosciences, Biomedicine, and Movement Sciences, Section of Biology and Genetics, University of Verona, Verona, Italy.

${ }^{2}$ Department of Surgery, Dentistry, Pediatrics and Gynaecology, Unit of Vascular and Endovascular Surgery, University of Verona, Verona, Italy.

${ }^{3}$ Department of Neurosciences, Biomedicine, and Movement Sciences, Section of Neurophatology, University of Verona, Verona, Italy.

${ }^{4}$ Centre for prevention of stroke and dementia Nuffield, Department of Clinical Neurosciences, University of Oxford.

\section{Corresponding author:}

Dr. Macarena Gomez-Lira

Department of Neurosciences, Biomedicine, and Movement Sciences, Section of Biology and Genetics, University of Verona, Verona, Italy. Strada Le Grazie, 8.

37134 Verona, Italy.

E-mail address: macarena.gomezlira@ univr.it

Keywords: Gene expression, blood and plaques, atherosclerosis.

Cover Title: TLR4-PTGS2 signaling in blood and carotid plaques 


\section{ABSTRACT}

Toll-like receptor 4 (TLR4)/Prostaglandine synthetase 2 (PTGS2) signaling plays a relevant role in atherosclerotic plaque vulnerability.

Aim: The purpose of this study was to check the gene expression of 6 genes participating to TLR4/PTGS2 signaling (TLR4, PTGS2, ACSL4, PTGER3, PTGER4, and EPRAP) in carotid plaques and blood samples from the same individual and to evaluate these genes as biomarker of plaque progression.

Methods: We investigated differential gene expression by qRT-PCR in 62 atherosclerotic patients' carotid plaques and corresponding blood sample.

Results: A very weak or no correlation was observed in the overall population or analyzing asymptomatic patients.

Conclusion: These analyzed genes are most likely not suitable for inclusion in the clinical routine as biomarkers of plaque instability. 


\section{INTRODUCTION}

Ischemic stroke is one of the major causes of disease worldwide, making its prevention one of the major public health challenges. Rupture of the atherosclerotic plaque surface and the subsequent formation of luminous thrombus are probably the most important mechanisms underlying the acute ischemic event. Atherosclerosis is a chronic disease characterized principally by lipid accumulation and inflammation. Plaque inflammation is believed to play a key role in plaque vulnerability and eventually, plaque rupture and thrombosis leading to ischemic events [1]

Regardless of the state-of-the-art cardio and cerebrovascular treatments, these diseases are the most common cause of death worldwide, indicating that important pathogenic mechanisms escape the actual treatment modalities. Persistent inflammation may represent one of those mechanisms. Considering the central role of inflammation, research on systemic markers of inflammation have been evaluated in asymptomatic as well as symptomatic patients, to predict the future risk of ischemic events and by assumption, the presence of high-risk plaques [2, 3].

Peripheral whole blood is frequently used as a surrogate tissue for gene expression analysis of human tissue that is not easily available. There is generally greater enthusiasm for the biomarker if the factor being measured is along a causal pathway that is reasonably associated with the vascular outcome. The underlying theory in such studies is that genes expressed in tissue leave a transcriptional footprint in blood. Although distant from atherosclerotic lesions, peripheral blood mononuclear cells including lymphocytes, monocytes, and granulocytes are easily accessible [4] and their gene expression may be altered in response to inflammatory cytokines released from atherosclerotic lesions.

Several high throughput studies have identified genes differentially expressed in blood of atherosclerotic patients [5], but these methods may not capture genes with moderate expression differences that might have important functional roles and could represent valid biomarkers of plaque progression. Recently, accumulating interest has focused on TLR4 involvement in atherosclerosis lesions during development and progression of atherosclerotic disease [6]. We have recently reported a diverse activation of TLR4/PTGS2 signaling between plaques of symptomatic and asymptomatic atherosclerotic patients [7], indicating that this signaling contributes to plaque vulnerability. It would be interesting to see if this different activation leaves a track in peripheral blood that could contribute to the identification of individuals at high risk of ischemic events.

The aim of the present study was to evaluate in the same patient, the concordance of expression values, if any, between specific genes participating in the TLR4/PTGS2 signaling (TLR4, PTGS2, 
ACSL4, PTGER3, PTGER4, and EPRAP) in atherosclerotic plaque and corresponding peripheral blood sample.

\section{MATERIAL AND METHODS}

\section{Study patients}

Consecutive eligible patients with internal carotid artery stenosis undergoing carotid endarterectomy at GB Rossi University Hospital and Civile Maggiore Hospital (Verona) were included in this study. Blood samples were obtained from each patient immediately prior to endarterectomy. For gene expression analysis, plaque and blood samples were obtained from 25 atherothrombotic stroke patients, and 37 patients presenting asymptomatic carotid plaques causing carotid stenosis of $60 \%$ or more, not associated with any neurological symptom (MRC ACST, 2004). The symptomatic group included patients who presented Transient Ischemic Attack (TIA) or ischemic stroke within the previous 23 days to endarterectomy. Atherothrombotic nature of stroke was established by i) neuroradiologic aspect of a non-lacunar lesion in the carotid artery territory; ii) evidence of ipsilateral cerebral embolization on transcranial Doppler monitoring; iii) no embolic cardiac sources as assessed by echocardiography; iv) ipsilateral carotid plaque which could benefit from carotid endarterectomy according to North American Symptomatic Carotid Endarterectomy Trial (NASCET) [8]. Information and characteristics of patients, vascular risk factors, and current therapy were collected by study neurologists. Exclusion criteria included intracerebral hemorrhage, inflammatory pathologies, and patients ongoing non-steroidal or glucocorticoid anti-inflammatory therapy. The study was approved by theEthical Committee of the Hospital and informed written consent was obtained from all patients before enrollment. Plaques analyzed in the present study were part of plaques described in a larger group of patients were the correlation between genes was reported [7].

\section{RNA extraction from whole blood}

Blood samples (2.5 mL) were collected in PAXgene Blood RNA tubes and total RNA was isolated using the standardized RNA Kit (PreAnalytiX, Qiagen).

\section{RNA extraction from carotid plaques}

Total RNA from the entire carotid plaques was purified by ultracentrifugation in cesium chloride gradient after homogenization in a $4 \mathrm{M}$ solution of guanidinium thiocyanate/ $\beta$ mercaptoethanol. Total RNA was quantified by absorbance at A260 nm and the purity was assessed by $\mathrm{A}_{260} \mathrm{~nm} / \mathrm{A}_{280}$ 
$\mathrm{nm}$ ratio. RNA integrity was confirmed by non-denaturing agarose gel electrophoresis. RNA was stored at $-80^{\circ}$ for later use as described [7]

\section{Reverse Transcription and Real-Time PCR}

RNA samples were reverse transcribed using a cDNA synthesis kit (Invitrogen). TLR4, PTGS2, ACSL4, EPRAP, PTGER3, and PTGER4 gene expression was determined by RealTime PCR using SYBR Green I as described by Ferronato et al., 2011 [9]. The specificity of the amplicons was determined by Sybr green fluorescence as a function of temperature (melting curve analysis). Amplification efficiency was calculated for each assay by a standard curve made out of four serial dilutions of a pool of cDNA of symptomatic and asymptomatic patients. The complete experiments were conducted twice. Glyceraldehyde-3-phosphate dehydrogenase (GAPDH) and TATA box binding protein (TBP) genes were chosen as the endogenous controls to normalize target genes. For accurate averaging of the control genes, the geometric mean was used instead of the arithmetic mean, as the former is more accurate for possible outlying values and abundance differences between the different genes [10]. Primers used for amplification are described by Ferronato et al., 2018 [7]. Comparative quantification of gene expression was determined using Pfaffl's efficiency corrected calculation.

\section{Statistical analysis}

Spearman correlation was conducted to investigate the correlation of gene expression between blood and plaques. Data were log-transformed when not normally distributed. Statistical analysis was done with logarithmically transformed values. Differences in gene expression were analyzed with GraphPad Prism statistical program, using non-parametric Mann-Whitney test. In terms of risk factors and drug therapy, associations between variables were evaluated by use of Fisher's exact test. The significance of the results was adjusted for multiple tests, giving significance at $\mathrm{P}$ value $\leq 0.01$.

\section{RESULTS}

Demographic and clinical data of subjects are summarized in Table 1. The asymptomatic group of patients was represented by 24 men and 13 women with a mean age of $72 \pm 8$. The symptomatic

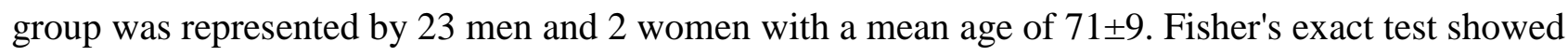
no significant differences in cardiovascular risk factors and current drug therapy between the two groups of patients. Sex was the only variable significantly different between groups $(\mathrm{P}<0.05)$, being women less represented in the symptomatic group of patients. 
Correlation of gene expression between blood and plaques from the same subject.

A very weak or no correlation was observed between blood and plaque gene expression (Figure 1), due to the difference observed in each individual patient. PTGER3 gene was not expressed in blood. The PTGS2 mRNA expression was higher in blood than in plaques in 38,7\% (24 samples), lower in $32,3 \%$ (20 samples), and in 29\% (18 samples) of patients expression values were comparable in plaque and in blood of patients, EP4 expression was higher in blood than in plaques in 27,42\% (17 pair samples), lower in 29\% (18 pair samples), and comparable in 43,6\% (27 paired samples) of patients, TLR4 expression was higher in $32,3 \%$ (20 paired samples), lower in $22,6 \%$ (14 paired samples), comparable in 45,2\% (28 paired samples) of patients, EPRAP expression was higher $33,9 \%$ (21 paired samples), lower in 24,2\% (15 paired samples), and comparable in 41,9\% (26 paired samples) of patients, and ACSL4 was higher in blood than in plaques in 30,6\% (19 paired samples), lower in $22,6 \%$ (14 paired samples), and comparable in 46,8\% (29 paired samples) of patients. EP3 gene was expressed in plaques but not in blood of any patient.

\section{Correlation of blood and plaques of stratified patients}

The same results were obtained stratifying by symptomatic and asymptomatic patients were likewise a very weak or no correspondence was observed (table 2).

We have previously reported that the evaluation of gene expression in blood of symptomatic and asymptomatic patients showed an upregulation of PTGS2 and TLR4 gene expression in symptomatic patients, and no difference in PTGER4 (EP4) gene expression between groups [9]. No other differences in gene expression between symptomatic and asymptomatic patients was observed as shown in Online Resource Figure 1.

\section{DISCUSSION}

Atherosclerosis is a major risk factor for stroke. Up to $15 \%$ of strokes and transient ischaemic attacks (TIA) are associated with severe carotid stenosis. Surgical treatment of carotid stenosis has proven to be superior to medical treatment alone in subgroups of patients with higher stroke risk. With new medical therapies, a consistent number of low-risk patients could benefit from medical treatment alone avoiding surgery, and patients with stenosis $\leq 60 \%$ could be at risk and be considered for surgical therapy. Hence, the possibility to stratify stroke risk in such patients is crucial for stroke therapy and prevention. Different approaches have been undertaken aiming to correlate non-invasive methodologies with vulnerable, prone to rupture plaques [11].

Given the evident heritability of many pathologies, numerous research has been concentrated on identifying genomic variations underlying susceptibility. These studies have been based on the 
notion that the genome is equal in sequence and structure in all cells and tissues of the same organism. This principle allows for the usual practice of examining DNA sequence variations in peripheral blood in relation to risk for diseases [12]. Different is when searching for gene expression in blood as marker of specific tissue disease. In fact, there is no clear consensus about the use of blood-based gene expression data for addressing research questions, since the expression of genes is mostly tissue-specific [13]. Nevertheless, gene expression in blood has emerged as a viable approach for the identification of biomarkers of disease [14-18], and it is worth to test if specific gene transcriptional activity in blood samples could provide biomarkers for atherosclerotic plaque vulnerability. We tested this theory by analyzing gene expression of the TLR4-PGE2 signaling in plaques and blood of symptomatic and asymptomatic patients. Many studies, specially referred to coronary artery disease, have evaluate gene expression in blood and tissue samples, and compared levels of gene expression in one with the other group [19-24], but information about gene expression in both, plaque and blood from the same individual is absent.

Aiming to investigate if the expression of six candidate genes participating in the TLR4-PGE2 signaling could contribute as markers for plaque vulnerability, we compared their expression in whole blood and corresponding carotid plaque of patients. However, all genes evaluated behaved differently in blood and plaques among patients. We also tested the possibility that our results could be influenced by the peripheral inflammatory response to stroke [25]. To avoid this eventual bias, we stratified patients by symptomatic and asymptomatic. However, as in the entire group, a very week or no correlation was observed between blood and plaques, indicating that these genes are unlikely to be good candidates as markers of plaque progression to vulnerability.

Our study has the strength of comparing blood and plaques of the same patient which avoid bias due to differences between patients genetic and environmental background. The limitation of this study could reside in the restricted number of patients that could lead to a type II error.

In conclusion, the current study indicates that the expression in blood of genes involved in the TLR4/PTGS2 signaling does not correlate with the expression in the atherosclerotic carotid plaques. Consequently, the expression of these genes is probably not suitable for inclusion in the clinical routine as biomarkers of plaque instability. 


\section{Compliance with Ethical Standards}

Disclosure of potential conflicts of interest:

Authors:

Silvia Ferronato declares to have no conflict of interest.

Alberto Scuro declares to have no conflict of interest.

Macarena Gomez-Lira declares to have no conflict of interest.

Sara Mazzucco declares to have no conflict of interest.

Elisa Olivato declares to have no conflict of interest.

Alberto Turco declares to have no conflict of interest.

Elisa Orlandi declares to have no conflict of interest.

Stefania Fochi declares to have no conflict of interest.

Maria Grazia Romanelli declares to have no conflict of interest.

Research Involving Human Participants and/or Animals:

The present study was performed in accordance with the ethical guidelines of the declaration of Helsinki and was approved by the Ethical Committee of the Hospital

Informed consent:

all patients were informed of the aim of the study and provided written consent. 


\section{REFERENCES}

1. Hansson GK, Libby P, Tabas I (2015) Inflammation and plaque vulnerability. J Intern Med 278, 483-93. doi: 10.1111/joim.12406.

2. Shah PK (2014) Biomarkers of Plaque Instability. Curr Cardiol Rep 16, 547. doi: $10.1007 / \mathrm{s} 11886-014-0547-7$.

3. Soeki T, Sata M (2016) Inflammatory Biomarkers and Atherosclerosis. Int Heart J 57, 134 139. doi: 10.1536/ihj.15-346.

4. Chávez-Sánchez L, Espinosa-Luna JE, Chávez-Rueda K et al (2014) Innate Immune System Cells in Atherosclerosis. Arch Med Res 45, 1-14. doi:

10.1016/j.arcmed.2013.11.007.

5. Chen HH, Stewart AFR (2016) Transcriptomic Signature of Atherosclerosis in the Peripheral Blood: Fact or Fiction? Curr Atheroscler Rep 18, 77.

6. Li H, Sun B (2007) Toll-like receptor 4 in atherosclerosis. J Cell Mol Med 11, 88-95.

7. Ferronato S, Scuro A, Gomez-Lira M et al (2018) Correlations between gene expression highlight a different activation of ACE/TLR4/PTGS2 signaling in symptomatic and asymptomatic plaques in atherosclerotic patients. Mol Biol Rep 1-6. doi: 10.1007/s11033018-4207-7.

8. Barnett HJM, Taylor DW, Eliasziw M et al (1998) Benefit of carotid endarterectomy in patients with symptomatic moderate or severe stenosis. North American Symptomatic Carotid Endarterectomy Trial Collaborators. N Engl J Med 339, 1415-1425.

9. Ferronato S, Gomez-Lira M, Olivato S et al (2011) Upregulated Expression of Toll-like Receptor 4 in Peripheral Blood of Ischaemic Stroke Patients Correlates with Cyclooxygenase 2 Expression. Eur J Vasc Endovasc Surg 41, 358-363. doi: 10.1016/j.ejvs.2010.11.019

10. Vandesompele J, De Preter K, Pattyn F et al (2002) Accurate normalization of real-time quantitative RT-PCR data by geometric averaging of multiple internal control genes. Genome Biol 3, RESEARCH0034.

11. Tavakoli S, Vashist A, Sadeghi MM (2014) Molecular imaging of plaque vulnerability. J Nucl Cardiol 21(6):1112-28. doi: 10.1007/s12350-014-9959-4.

12. Titov BV, Osmak GJ, Matveeva NA, et al (2017) Genetic risk factors for myocardial infarction more clearly manifest for early age of first onset. Mol Biol Rep 44(4):315-321. 
doi: 10.1007/s11033-017-4112-5.

13. Greene CS, Krishnan A, Wong AK et al (2015) Understanding multicellular function and disease with human tissue-specific networks. Nat Genet 47, 569-76. doi: 10.1038/ng.3259.

14. Munkholm K, Peijs L, Vinberg M et al (2015) A composite peripheral blood gene expression measure as a potential diagnostic biomarker in bipolar disorder. Transl Psychiatry, 5:e614.

15. Ponnampalam SN, Kamaluddin NR, Zakaria Z et al (2017) A blood-based gene expression and signaling pathway analysis to differentiate between high and low grade gliomas. Oncol Rep, 37:10-22.

16. Johnson RH, Hu P, Fan C et al (2015) Gene expression in "young adult type" breast cancer: a retrospective analysis. Oncotarget, 6:13688-702.

17. Long F, Su J-H, Liang B et al (2015) Identification of Gene Biomarkers for Distinguishing Small-Cell Lung Cancer from Non-Small-Cell Lung Cancer Using a Network-Based Approach. Biomed Res Int, 2015:1-8.

18. Wu C, Liu C, Luo K et al (2018) Changes in Expression of the Membrane Receptors CD14, MHC-II, SR-A, and TLR4 in Tissue-Specific Monocytes/Macrophages Following Porphyromonas gingivalis-LPS Stimulation. Inflammation, 41:418-431.

19. Sinnaeve PR, Donahue MP, Grass P et al (2009) Gene expression patterns in peripheral blood correlate with the extent of coronary artery disease. PLoS One 4, e7037. doi: 10.1371/journal.pone.0007037.

20. Abdullah MHN, Othman Z, Noor HM et al (2012) Peripheral blood gene expression profile of atherosclerotic coronary artery disease in patients of different ethnicity in Malaysia. $\mathbf{J}$ Cardiol 60, 192-203. doi: 10.1016/j.jjcc.2012.05.009.

21. Voros S, Elashoff MR, Wingrove JA et al (2014) A peripheral blood gene expression score is associated with atherosclerotic Plaque Burden and Stenosis by cardiovascular CTangiography: Results from the PREDICT and COMPASS studies. Atherosclerosis 233, 284-290. doi: 10.1016/j.atherosclerosis.2013.12.045.

22. Fan Z, Ji H, Li Y et al (2017) Relationship between monocyte-to-lymphocyte ratio and coronary plaque vulnerability in patients with stable angina. Biomark Med. 11, 979-990. doi: 10.2217/bmm-2017-0235.

23. Arapi B, Bayoğlu B, Cengiz M et al (2018) Increased Expression of Interleukin-18 mRNA 
is Associated with Carotid Artery Stenosis. Balkan Med . 35, 250-255. doi:

10.4274/balkanmedj.2017.0323.

24. Kim SH, Kim GJ, Umemura T et al (2017) Aberrant expression of plasma microRNA-33a in an atherosclerosis-risk group. Mol Biol Rep. 44(1):79-88. doi: 10.1007/s11033-0164082-z.

25. Maas MB, Furie KL (2009) Molecular biomarkers in stroke diagnosis and prognosis. Biomark Med. 3, 363-383. doi: 10.2217/bmm.09.30. 